\title{
An Avid Imitator
}

\author{
Dimitrios Farmakiotis ${ }^{\mathrm{a}, \mathrm{b}, \mathrm{d}}$, Alexis Liakos ${ }^{\mathrm{a}, \mathrm{d}}$, Michael B. Miller ${ }^{\mathrm{c}}$, Jeffrey F. Krane ${ }^{\mathrm{c}}$, \\ Lindsey R. Baden ${ }^{\mathrm{a}}$, Sarah P. Hammond ${ }^{\mathrm{a}}$
}

\begin{abstract}
We present a case of disseminated cryptococcal disease, coexisting with and mimicking lymphoma. Determination of serum cryptococcal antigen should be considered for lymphopenic patients with hematologic malignancies, presenting with unexplained fever, and/or lymphadenopathy and/or pulmonary findings. Patients with hematologic malignancies treated with chemotherapy regimens are susceptible to diverse opportunistic infections. Therefore, in this patient population, it is often necessary to obtain a definitive pathologic diagnosis, to diagnose uncommon syndromes and guide management.
\end{abstract}

Keywords: Cryptococcus; Lymphoma; Opportunistic infections

\section{Introduction}

In patients with hematologic malignancies (HMs), disease progression or relapse is frequently the most common consideration in the differential diagnosis of clinical syndromes with systemic symptoms and signs. However, patients with HM, especially those treated with intensive chemotherapy regimens, are susceptible to opportunistic bacterial, viral, but also fungal infections, including cryptococcal disease [1-3]. Herein, we present an unusual case of disseminated cryptococcosis, coexisting with and mimicking lymphoma.

\section{Case Report}

A 57-year-old man with non-Hodgkin's lymphoma presented

Manuscript accepted for publication January 06, 2016

aDivision of Infectious Diseases, Brigham and Women's Hospital and DanaFarber Cancer Institute Harvard Medical School, Boston, MA, USA

${ }^{b}$ Division of Infectious Diseases, Warren Alpert Medical School of Brown University, Providence, RI, USA

'Department of Pathology, Brigham and Women's Hospital and Dana-Farber Cancer Institute Harvard Medical School, Boston, MA, USA

${ }^{\mathrm{d} C}$ Corresponding Author: Dimitrios Farmakiotis, 593 Eddy Street, Gerry House 113, Providence, RI 02903, USA. Email: dimitrios.farmakiotis@lifespan.org; Alexis Liakos, Brigham and Women's Hospital, PBB-A4, 75 Francis St, Boston, MA 02115, USA. Email: aliakos@partners.org

doi: http://dx.doi.org/10.14740/jocmr2458w to another institution 4 days after his third cycle of rituximab, cyclophosphamide, adriamycin, vincristine and prednisone (R-CHOP) with headache, malaise and fevers up to $102^{\circ} \mathrm{F}$. A chest computerized tomography (CT) showed a right upper lobe opacity concerning for pneumonia. He received 11 days of empiric vancomycin, cefepime and doxycycline for tickborne diseases and was discharged home to complete an additional 7-day course of levofloxacin and doxycycline. He continued to feel unwell and was readmitted to our institution the following day with malaise and tachycardia. His past medical history was significant for follicular lymphoma, localized below the diaphragm with extra-nodal (lumbar spine) involvement diagnosed 4 months prior to presentation, hypertension and pulmonary embolism. He worked as a computer programmer and had not traveled outside of New England. He had no history of animal exposures. His vital signs on admission were: temperature $100.5^{\circ} \mathrm{F}$, blood pressure $130 / 90 \mathrm{~mm} \mathrm{Hg}$, heart rate $130 / \mathrm{min}$, respiratory rate $18 / \mathrm{min}$, and oxygen saturation $98 \%$ on room air; he was not in distress. He had no peripheral lymphadenopathy or skin lesions. His cardiovascular, pulmonary, abdominal and neurologic exams were unremarkable. His laboratory workup was notable for lymphopenia (absolute lymphocyte count of 250 cells $/ \mu \mathrm{L}$ ) and hyponatremia (125 mEq/L). Blood, urine and sputum cultures were negative. Positron emission tomography/CT (PET/CT) performed to evaluate for lymphoma progression showed enlarged, fluoro-2-deoxy-D-glucose (FDG)-avid mediastinal lymphadenopathy, which was new compared to 3 months prior, and had a maximum standardized uptake value (SUV) of 7, suggestive of active lymphoma (Fig. 1A). A bronchoscopy with ultrasound-guided fine-needle aspiration of a mediastinal lymph node was performed.

Hematoxylin-eosin (Fig. 1B) and mucicarmine (Fig. 1C) stains of an endobronchial ultrasound-guided fine-needle aspirate from the lymph node revealed many encapsulated yeast forms with narrow-based budding, morphologically consistent with Cryptococcus species, and no malignant cells. Serum cryptococcal antigen titer was 1:128. CD4 ${ }^{+}$lymphocyte count was 78 cells $/ \mu \mathrm{L}$ and he was HIV negative. Although the headache that he initially presented with had resolved, a lumbar puncture was performed [1]. The opening pressure was $28 \mathrm{~mm}$ $\mathrm{Hg}$. The cerebrospinal fluid (CSF) white blood cell count was 365 cells $/ \mu \mathrm{L}, 87 \%$ of which were neutrophils, and protein was elevated (416 mg/dL, normal range: 40 - $70 \mathrm{mg} / \mathrm{dL})$. Cryptococcal antigen in the CSF was also positive (1:128), and CSF cultures grew Cryptococcus neoformans.

Our patient received induction treatment with liposomal amphotericin-B (5 mg/kg daily) and 5-flucytosine $(100 \mathrm{mg} / \mathrm{kg}$ 


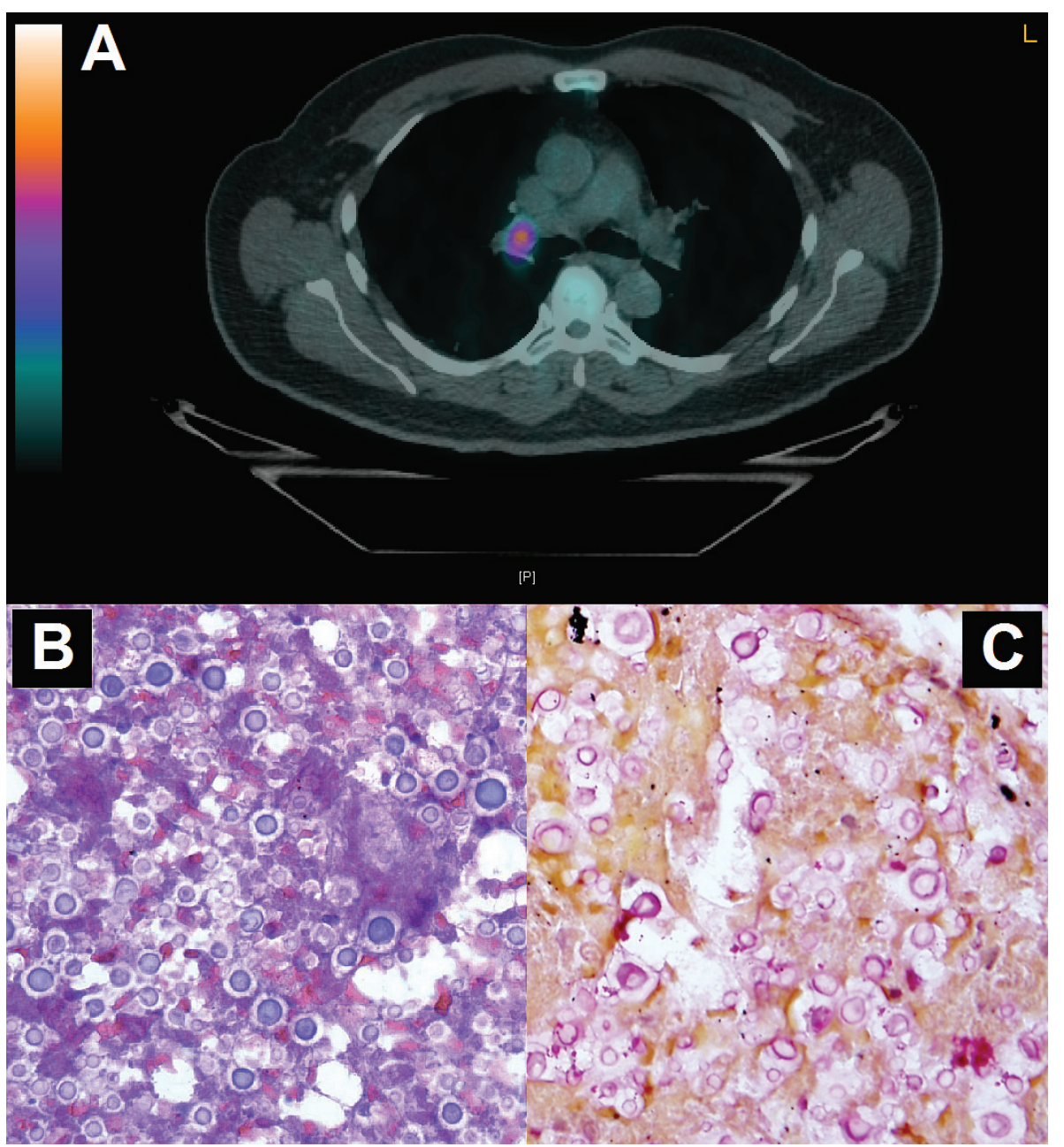

Figure 1. (A) PET/CT showing enlarged, FDG-avid mediastinal lymphadenopathy. (B) Hematoxylin-eosin and (C) mucicarmine stains of an endobronchial ultrasound-guided fine-needle aspirate from the mediastinal lymph node showing many encapsulated yeast forms with narrow-based budding, morphologically consistent with Cryptococcus species.

daily) for 2 weeks [1]. His symptoms resolved, repeat CSF cultures were negative, and he transitioned to oral fluconazole $800 \mathrm{mg}$ daily for 8 weeks, and $400 \mathrm{mg}$ daily until completion of chemotherapy, with the plan to continue $200 \mathrm{mg}$ of fluconazole daily (maintenance) indefinitely, at least until lymphocyte count recovery [1].

\section{Discussion}

Between 1956 and 1972, the frequency of cryptococcal infection in patients with chronic lymphocytic leukemia and Hodgkin's lymphoma was estimated to be 24.3 and 13.3 per 1,000 patients, respectively [2]. However, in a more recent cohort [3], the incidence of cryptococcosis at a large cancer center was 18/100,000 admissions, between 1989 and 1999. Furthermore, infectious lymphadenitis was observed in $3.9 \%$ of lymph node biopsies among patients with chronic lymphocytic leukemia and small-cell lymphoma at one center between 2003 and 2012, but only one case was due to Cryptococcus neoformans
[4]. The lower rates of documented cryptococcal disease recently may be related to increased use of azole prophylaxis in cancer patients who are at risk for invasive fungal infections, particularly those with HM.

Cryptococcosis typically affects patients who have impaired cell-mediated immunity or receive high-dose steroids $[3,5-8]$. In two case series of cancer patients with proven cryptococcal infection, $83 \%$ [2] and 65\% [3] had lymphoproliferative disorders, most of whom had advanced cancer and were heavily treated. We know little about the risk for cryptococcosis in presumably lower net states of immunosuppression, for example with low-intensity chemotherapy, or early in the course of lymphoma treatment. In the two studies mentioned above, $100 \%$ [2] and 61\% [3] of affected patients had significant lymphopenia, an important risk factor for cryptococcal disease, as exemplified in the present and previous case reports [5-7].

The clinical and radiological presentation of cryptococcosis in cancer patients is non-specific and frequently mimics progression of primary malignancy or metastatic disease [3, 
$5,7,9]$. In one study of cancer patients with cryptococcal disease, pulmonary cryptococcosis was initially misdiagnosed as cancer in $35 \%$, healthcare-associated pneumonia in $22 \%$, and indeterminate lung nodules in 19\% [3]. 18-FDG PET/CT is often used to differentiate between benign and malignant lesions, and an SUV of approximately 5 is typically considered a reliable cutoff [5]. Nevertheless, in our patient, cryptococcal involvement of lymph nodes manifested with an SUV of 7, and in another similar case with an SUV of 13 [5]. In a series of seven patients with pulmonary cryptococcal lesions, SUV values ranged between 2.2 and 11.6 [9]. It should be noted that clinical and radiological responses to chemotherapy observed in other lymphoma areas usually favor infectious causes of new FDG-avid lesions.

Immunocompromised patients may have atypical clinical and radiographic presentations of infectious diseases, so pathologic confirmation is often necessary to make a correct diagnosis. As this case demonstrates, cytologic sampling can readily demonstrate the presence of fungal organisms, without the delays involved with special stains and/or fungal cultures. Accurate diagnosis is particularly important for targeted antifungal treatment, given the emerging threat of resistance among medically important fungi, as a result of increased empiric and prophylactic use of antifungal agents [10].

In conclusion, cryptococcal disease can coexist with and mimic lymphoma. Determination of serum cryptococcal antigen should be considered for lymphopenic patients with unexplained fever, lymphadenopathy, and pulmonary findings. The present report highlights the importance of obtaining a definitive pathologic diagnosis to diagnose uncommon syndromes and guide treatment in patients with hematologic malignancies.

\section{References}

1. Perfect JR, Dismukes WE, Dromer F, Goldman DL, Graybill JR, Hamill RJ, Harrison TS, et al. Clinical prac- tice guidelines for the management of cryptococcal disease: 2010 update by the infectious diseases society of america. Clin Infect Dis. 2010;50(3):291-322.

2. Kaplan MH, Rosen PP, Armstrong D. Cryptococcosis in a cancer hospital: clinical and pathological correlates in forty-six patients. Cancer. 1977;39(5):2265-2274.

3. Kontoyiannis DP, Peitsch WK, Reddy BT, Whimbey EE, Han XY, Bodey GP, Rolston KV. Cryptococcosis in patients with cancer. Clin Infect Dis. 2001;32(11):E145150.

4. Bowen DA, Rabe KG, Schwager SM, Slager SL, Call TG, Viswanatha DS, Zent CS. Infectious lymphadenitis in patients with chronic lymphocytic leukemia/small lymphocytic lymphoma: a rare, but important, complication. Leuk Lymphoma. 2015;56(2):311-314.

5. Hamerschlak N, Pasternak J, Wagner J, Perini GF. Not all that shines is cancer: pulmonary cryptococcosis mimicking lymphoma in [(18)] F fluoro-2-deoxy-D-glucose positron emission tomography. Einstein (Sao Paulo). 2012;10(4):502-504.

6. Marchand T, Revest M, Tattevin P, Chevrier S, Poullot E, Lamy T, Houot R. Early cryptococcal meningitis following treatment with rituximab, fludarabine and cyclophosphamide in a patient with chronic lymphocytic leukemia. Leuk Lymphoma. 2013;54(3):643-645.

7. Slavin MA, Chen SC. Cryptococcosis, lymphoproliferative disorders and modern day chemotherapy regimens. Leuk Lymphoma. 2013;54(3):449-450.

8. Liapis K, Taussig D, Cotter FE, Gribben JG. Cutaneous cryptococcosis in Hodgkin lymphoma. Br J Haematol. 2014;164(4):467.

9. Huang CJ, You DL, Lee PI, Hsu LH, Liu CC, Shih CS, Shih CC, et al. Characteristics of integrated 18F-FDG PET/CT in Pulmonary Cryptococcosis. Acta Radiol. 2009;50(4):374-378.

10. Farmakiotis D, Tarrand JJ, Kontoyiannis DP. Drugresistant Candida glabrata infection in cancer patients. Emerg Infect Dis. 2014;20(11):1833-1840. 